\title{
Maternal and perinatal outcomes in diabetic pregnancies at BPKIHS
}

\author{
Anamika Das* \\ Department of Obstetrics and Gynecology, B. P. Koirala Institute of Health Sciences, Dharan, Nepal
}

Received: 06 April 2017

Accepted: 02 May 2017

\section{*Correspondence:}

Dr. Anamika Das,

E-mail: dranamikadas123@gmail.com

Copyright: $\odot$ the author(s), publisher and licensee Medip Academy. This is an open-access article distributed under the terms of the Creative Commons Attribution Non-Commercial License, which permits unrestricted non-commercial use, distribution, and reproduction in any medium, provided the original work is properly cited.

\begin{abstract}
Background: Diabetes has become a global pandemic because of aging population, sedentary life style, urbanization, and increasing incidence of obesity; this study was aimed to identify the maternal and perinatal outcome in Diabetes complicating pregnancies and to compare the outcome in Gestational Diabetes Mellitus and Overt Diabetes Mellitus.

Methods: This was a descriptive study conducted from $1^{\text {st }}$ April 2013-31 $1^{\text {st }}$ March 2014 on total 75 pregnant women. All antenatally diagnosed cases of GDM and overt diabetes, booked or unbooked, whoever came for delivery were studied. Both mothers and neonates were followed up in ante-partum, intra-partum, and post-partum period till their stay in hospital. Management of the patient was done as per the hospital protocol.

Results: Out of 75 cases enrolled, $25.33 \%$ had GDM and 16\% had overt diabetes mellitus.57.33\%patients were multigravida, among which $40 \%$ had GDM and $17.33 \%$ had Overt Diabetes Mellitus.61.33\% patients underwent LSCS, of which $41.33 \%$ had GDM and $20 \%$ had overt diabetes mellitus. Obstetric complication was more common in GDM patients (30.66\%) compared to Overt (22.66\%). 89.33\%babies had good neonatal outcome and $10.66 \%$ babies (6.66\% of GDM mothers and $4 \%$ of overt mothers) had poor neonatal outcome (macrosomia, hypoglycaemia, prematurity, respiratory distress with NICU stay and use of antibiotics, poor APGAR ( $<7$ at $5 \mathrm{~min}$ ), gross congenital malformation, hyperbilirubinemia, stillbirth and neonatal death).

Conclusions: Maternal and perinatal complications was commonly seen in diabetic pregnancy though more common in GDM.The time has come for the Government of Nepal to implement an effective plan to the benefit of pregnant women with diabetes during pregnancy.
\end{abstract}

Keywords: Caesarean section, Gestational diabetes mellitus, Macrosomia, Maternal and perinatal outcomes

\section{INTRODUCTION}

Gestational diabetes mellitus is defined as any degree of impaired glucose tolerance of with onset or first recognition during pregnancy. Overt Diabetes Mellitus is defined as women with a random plasma glucose level greater than $200 \mathrm{mg} / \mathrm{dl}$ plus classic signs and symptoms such as polydipsia, polyuria and unexplained weight loss or a fasting glucose exceeding $125 \mathrm{mg} / \mathrm{dl}$ are considered by the ADA (2004) to have overt diabetes. Nepal is a developing country and life style of many people has changed over one to two decades from active village farmer to sedentary city dweller. The prevalence of diabetes is increasing day by day in Nepal may be due to urbanization. The precise mechanisms underlying gestational diabetes remain unknown. The hallmark of GDM is increased insulin resistance. Pregnancy hormones and other factors are thought to interfere with the action of insulin as it binds to the insulin receptor. ${ }^{1}$ Pregnancy loss is significantly higher among women with diabetes compared to the non-diabeticpopulation. ${ }^{2}$ The effect of hyperglycaemia on the pregnancy outcome varies with the level of maternal blood glucose and the time during pregnancy with uncontrolled 
hyperglycaemia. Hyperglycaemia occurring early in pregnancy and during organogenesis as in the case of Overt DM, is associated with risk of congenital malformation, macrosomia, stillbirth, birth asphyxia and preterm delivery, while the same complications might appear with GDM but less frequently and less severe due to late occurrence of hyperglycaemia. So, Pregnancy related morbidity and mortality in gestational diabetes is less than that of overt diabetes mellitus however if not treated it is significantly higher than for non-diabetic women. Neonatal mortality is also higher among infants of diabetic mothers in approximately fifteen-fold when compared to the general population. ${ }^{3}$

The aim of this study is to compare the maternal and perinatal outcome in GDM and overt DM.

\section{METHODS}

This was a descriptive study done over 12 months from $1^{\text {st }}$ April 2013-31 $1^{\text {st }}$ March 2014). It was done in the antenatal ward of the Department of Obstetrics and Gynaecology, B. P. Koirala Institute of Health Sciences, Dharan. The study was done only after approval by Institutional Ethical Review Board. For sample size calculation, all the pregnant patients who met the inclusion criteria during study period were taken as cases $(n=75)$ based on hospital record of previous year.

The inclusion criteria included all antenatally diagnosed cases of GDM and overt diabetes, booked or unbooked, whoever came for delivery were studied. Both mothers and neonates were followed up in ante-partum, intrapartum, and post-partum period till their stay in hospital. Management of the patient was done as per the hospital protocol. Exclusion criteria were patients suffering from some other disorders which directly or indirectly may affect the outcome of pregnancy such as patient on corticosteroid therapy, Epilepsy, known hypertension, thyroid dysfunction. Written consent was taken before enrolling the patients. At admission, detailed history, general physical examination, abdominal examination and pelvic examination were done. Non-stress test was done after admission for fetal assessment. Patients were managed according to the standard management protocol followed in the hospital after explaining the maternal and fetal prognosis. Both the mother and baby were followed up till discharge after delivery for outcomes. Maternal variables assessed were age, gravidity, parity, gestational age at delivery, mode of delivery, Neonatal outcomes included birth weight, macrosomia (birth weight $>4 \mathrm{~kg}$ ) and rate of APGAR score less than 7 at $5 \mathrm{~min}$.

\section{Statistical analysis}

Corrected data were validated manually and enter it in MS-EXCEL 2007 and converted it into SPSS version 11.5 for statistical analysis Descriptive statistics were calculated like percentage, mean and standard deviation. The results were mentioned as graphical and tabular presentations. For inferential statistics, chi- square test were applied to find out the significant differences between GDM and Overt DM patients at $95 \%$ confidence interval and $80 \%$ power where $p$ value less that 0.05 was statically significant.

\section{RESULTS}

During the study period (one year), the total number of hospital delivery was 12,009 , amongst them total number of diabetic pregnancies was 75. Therefore, the hospital prevalence of diabetic pregnancy was seen to be $0.624 \%$. In this study, out of 75 diabetic cases, $n=43$ (57.33\%) was GDM and $n=32(42.66 \%)$ was overt diabetes. Diabetic pregnancies were less common in elderly mothers. Only $2.66 \%$ of diabetic admissions were in age group $>35$ years of which $1.33 \%$ had GDM and overt diabetes each which is not statistically significant $(\mathrm{p}=0.831)$. It was found that majority $(57.33 \%)$ of diabetic admissions were multigravida among which $40 \%$ had GDM and $17.33 \%$ had overt diabetes mellitus, the difference is statistically significant $(\mathrm{p}=0.012)$.

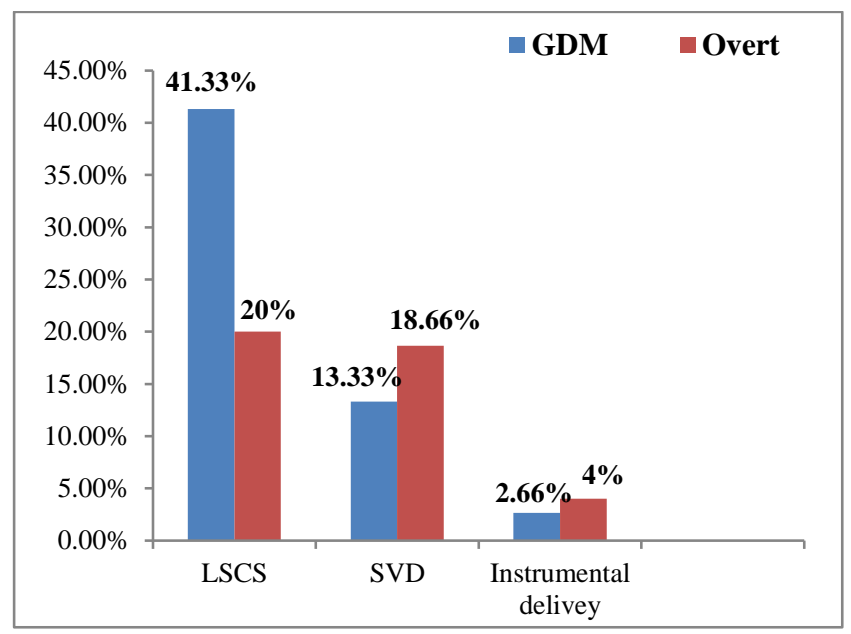

Figure 1: Mode of delivery in GDM and overt patients.

Majority of patients $(60 \%)$ got admitted for delivery at term POG (37-41 weeks), of which $40 \%$ had GDM and $20 \%$ had overt diabetes mellitus, the difference is statistically significant $(\mathrm{p}=0.045)$. Maximum women $(81.33 \%)$ had normal HbA1c (4.5-6.6\%) of which $40 \%$ had GDM and $33.33 \%$ had overt diabetes, the difference is not statistically significant $(\mathrm{p}=0.538)$.

The overall caesarean section rate was (61.33\%) ( $p=0.027$ ) of which $41.33 \%$ had GDM and $20 \%$ had overt diabetes mellitus and the most common indication for caesarean section was hypertension complicating pregnancy (Figure 1). Poor neonatal outcome was considered if neonates having macrosomia, hypoglycaemia, prematurity, respiratory distress leading to NICU stay and use of antibiotics, poor APGAR $(<7$ at $5 \mathrm{~min})$, gross congenital malformation, hyperbilirubinemia, stillbirth and neonatal death. 
Large for gestational age comprised nearly $9.33 \%$ patients of which $2.665 \%$ had GDM and $6.665 \%$ had overt diabetes, the difference is not statistically significant. It was observed that among 75 diabetic pregnancies in 43 GDM cases 42 neonates $(97.67 \%)$ were live at birth. There was one neonatal death in GDM.

Table 1: Distribution of mothers according to outcome among GDM and overt diabetes.

\begin{tabular}{|lll|}
\hline Characteristics & Maternal outcome & \\
\hline Preterm birth & GDM & Overt \\
\hline PROM & $15(20 \%)$ & $11(14.66 \%)$ \\
\hline Preterm Labour & $9(12 \%)$ & $6(8 \%)$ \\
\hline UTI & $4(5.33 \%)$ & $5(6.66 \%)$ \\
\hline Vulvovaginal candidiasis & $4(5.33 \%)$ & $3(4 \%)$ \\
\hline Preeclampsia & $2(2.66 \%)$ & $4(5.33 \%)$ \\
\hline Polyhydramnios & $2(2.66 \%)$ & $1(1.33 \%)$ \\
\hline Post-partum complications & $2(2.66 \%)$ & $0(0.0 \%)$ \\
\hline PPH & & $6(13.95 \%)$ \\
\hline Lactational failure & $8(18.60 \%)$ & $4(12.5 \%)$ \\
\hline Puerperal sepsis & $4(9.30 \%)$ & $4(12.5 \%)$ \\
\hline Prolonged labour & $3(6.97 \%)$ & $4(12.5 \%)$ \\
\hline
\end{tabular}

Table 2: Distribution of neonates according to outcome among GDM and overt diabetes

\begin{tabular}{|c|c|c|c|c|c|c|}
\hline \multirow{2}{*}{ Characteristics } & \multirow{2}{*}{ Category } & \multicolumn{2}{|c|}{ Neonatal Outcome } & \multirow[t]{2}{*}{ Total } & \multirow{2}{*}{$\begin{array}{l}\text { P- } \\
\text { Value }\end{array}$} & \multirow{2}{*}{ Remarks } \\
\hline & & GDM & Overt & & & \\
\hline \multirow{2}{*}{ Neonatal outcome } & Good & $38(50.66 \%)$ & $29(38.66 \%)$ & $67(89.33 \%)$ & \multirow{2}{*}{0.98} & \multirow{3}{*}{ NS } \\
\hline & Poor & $5(6.66 \%)$ & $3(4 \%)$ & $8(10.66 \%)$ & & \\
\hline Total & & 43 & 32 & 75 & & \\
\hline \multirow{3}{*}{ Birth Weight } & $<2.5$ & $2(2.66 \%)$ & $3(4 \%)$ & $5(6.66 \%)$ & \multirow{3}{*}{0.306} & \multirow{4}{*}{ NS } \\
\hline & $2.5-4$ & $39(52 \%)$ & $25(33.33 \%)$ & $64(85.33 \%)$ & & \\
\hline & $>4$ & $2(2.66 \%)$ & $4(5.33 \%)$ & $6(8 \%)$ & & \\
\hline Total & & 43 & 32 & 75 & & \\
\hline \multirow{3}{*}{ AGA/LGA } & AGA & $41(54.66 \%)$ & $27(36 \%)$ & $68(90.66 \%)$ & \multirow{3}{*}{0.106} & \multirow{3}{*}{ NS } \\
\hline & LGA & $2(2.66 \%)$ & $5(6.66 \%)$ & $7(9.33 \%)$ & & \\
\hline & & 43 & 32 & 75 & & \\
\hline \multirow{2}{*}{ Apgar Score } & $<7$ & $8(10.66 \%)$ & $3(4 \%)$ & $11(14.66 \%)$ & \multirow{2}{*}{0.264} & \multirow{3}{*}{ NS } \\
\hline & $>7$ & $35(46.66 \%)$ & $29(38.66 \%)$ & $64(85.33 \%)$ & & \\
\hline Total & & 43 & 32 & 75 & & \\
\hline
\end{tabular}

Further analysis showed that $85.33 \%$ neonates had APGAR score $>7$. Of which $46.66 \%$ had GDM and $38.66 \%$ had overt diabetes. $11.33 \%$ neonates had poor APGAR score $<7$ of which $10.66 \%$ had GDM and $4 \%$ had overt diabetes as depicted in Table 2.

\section{DISCUSSION}

In the present study, the prevalence of GDM increases with age from $10.67 \%$ in age group below 25 years to $25.33 \%$ in age group 25-30 years. Similarly, the prevalence in Overt DM also increases with age from $9.33 \%$ at <25years to $16 \%$ at $25-30$ years which is consistent with the study done by Seshiahet al which showed that prevalence of GDM increases with age from $14.5 \%$ in $15-19$ years to $25 \%$ in $\geq 30$-year-old age group.
Besides this, prevalence of GDM increased with gravidity. ${ }^{4}$ Seshiah et al study observed that GDM was increased with gravidity $(16.3 \%$ in primigravida and $25.8 \%$ in multigravida) and in this study also similar findings were noted where in GDM multigravida were at higher risk to develop GDM in comparison to primigravidaie. $^{4} \quad 40 \% \quad(\mathrm{n}=30)$ and $17.33 \% \quad(\mathrm{n}=10)$ respectively but this is not statistically significant.

A study conducted by Khan R et al showed Glycosylated haemoglobin was significantly higher in gestational diabetes than in controls. ${ }^{6}$ An early diagnosis of gestational diabetes provides safe baby and motherhood, and in particular, it will reduce the severity of complications and mainly foetal and maternal morbidity and mortality. In present study, it was found out that 
about $81.33 \%$ diabetic women had normal glycosylated haemoglobin, very few women $(8 \%)$ had higher BMI.A good glycaemic control was associated with good maternal and perinatal outcome.

There is a high incidence of operative delivery rate in women in GDM. In the current study, it was found that increased operative delivery was observed in GDM than overt cases. Those with GDM, $41.33 \%$ cases underwent LSCS and $13.33 \%$ cases delivered vaginally. Likewise, in Overt patients, $20 \%$ cases had LSCS delivery and $18.66 \%$ cases progressed spontaneously and delivered vaginally. Majority of caesarean deliveries performed in GDM cases were due to hypertension complicating pregnancy, previous LSCS and APH which is consistent with the study done by Deerochanawong et al in their study of 709 women, found that $32 \%$ and $70 \%$ of those diagnosed as GDM by WHO criteria and NDDG criteria underwent LSCS which is also supported by above studies. $^{6}$

In present study, $59.1 \%(n=13)$ of GDM cases and $40.9 \%$ $(n=9)$ in overt had features of preeclampsia which are insignificant statistically $(p>0.05)$. The high body mass index or obesity of women with gestational diabetes predisposed them to hypertension. In study by Deerochanawong et al, it was observed that $50 \%$ of GDM diagnosed by NDDG criteria and $12 \%$ of GDM diagnosed by WHO criteria had preeclampsia. ${ }^{6}$ Schmidt et al commented that $5 \%$ of GDM case diagnosed by WHO or ADA criteria had preeclampsia. ${ }^{7}$ They observed that GDM was associated with a 2-3-fold greater risk of preeclampsia. A study done by Sahu et al also favoured that preeclampsia was seen in $8 \%$ of GDM cases diagnosed by WHO criteria.

In this study, 1 case of GDM and 1 case in overt DM had developed polyhydramnios during, antenatal period which was statistically significant $(\mathrm{p}<0.05)$.

In a study by Sahu et al, polyhydramnios was most common antenatal complication and it was observed that $36 \%$ of GDM cases diagnosed by ADA and 22\% GDM cases diagnosed by WHO had polyhydramnios.

In this study, $4.65 \%(\mathrm{n}=2$ out of 43 ) neonates delivered from GDM cases having macrosomia and $12.5 \%(n=4$ out of 32) was reported in overt cases. It reflects that overt patients have more macrosomia as compare to GDM patients. In GDM, $(n=1)$ neonates born had cardiac malformation (PDA) and was referred to higher centre for needful, which was statistically insignificant $(\mathrm{p}>0.05)$.

As compared to neonates of GDM, no malformations were noted from overt neonates. Intrauterine death occurred in only one case of GDM, no intrauterine demise noted among overt cases. No perinatal mortality was observed in Overt cases but among GDM patients, $(n=1)$ perinatal mortality were occurred due to birth injury (shoulder dystocia) which was statistically insignificant.

Odar E et al observed that the babies of mothers with GDM (WHO criteria) were more likely to be macrosomic (36.7\%), perinatal mortality $(16.7 \%)$ and have shoulder dystocia $(23.3 \%)$ than those of normal mothers. ${ }^{8}$

In GDM group, hypoglycaemia was detected in one new born, 1 had polycythaemia, 1 developed birth injury (shoulder dystocia) leading to neonatal death, 1 had cardiac malformation (PDA) and 1 had neonatal jaundice. In Overt diabetes, two neonates developed RDS and 1 neonate developed neonatal sepsis. While comparing both groups, it was observed that GDM cases had more adverse neonatal outcome in comparison to overt diabetes.

Crowther et al in his study of Treatment of mild gestational diabetes vs no treatment, he found hypoglycaemia, hyperbilirubinemia, respiratory distress, and NICU admission were found respectively as $7 \%$ and $4 \%, 9 \%$ and $9 \%, 5 \%$ and $4 \%$, and $71 \%$ and $61 \%$ in both intervention and control group. ${ }^{9}$ A study done at Parkland Hospital, Dallas, Texas, risk of malformed infants delivered were of $1.5 \%$ of non-diabetic women and this risk was increased 3.2 fold $(4.8 \%)$ in women with gestational diabetes and the risk increased more that is about four fold $(6.0 \%){ }^{9}$

Study population was relatively small for the estimation of risk of congenital anomalies in babies of diabetic mothers; the study provides interesting information which can be helpful in planning maternal and child health services. In conclusion, as compared to non-diabetics, gestational diabetics have higher maternal and neonatal complications. With the availability of early prenatal detection and good antenatal care provided to these patients, one can expect to bring a perceptible improvement in the outcome of these pregnancies. The observation and quantification of maternal outcomes with GDM are necessary so that appropriate measures can be taken to reduce complications during pregnancy, delivery, and the neonatal period.

\section{CONCLUSION}

In conclusion, this study demonstrates that the prevalence of diabetic pregnancy was $0.624 \%$. Maternal complications were more common in GDM $(57.5 \%)$ than overt. Majority of neonates in diabetic pregnancy had good neonatal outcome, very few neonates had poor neonatal outcome. In terms of poor neonatal outcome, adverse neonatal outcome was more common in GDM as compared to overt DM.

In Nepal, there are no specialized programmes for diabetic pregnancy prevention with prenatal counselling, early detection of complications and proper management of diabetic pregnancies is lacking. Lack of awareness, 
illiteracy and difficult transportation further complicates the problem. Service is limited only in urban teaching institution.

So, the time has come for the Government of Nepal to implement an effective plan to the benefit of pregnant women with diabetes during pregnancy.

\section{ACKNOWLEDGMENTS}

Authors would like to thank Dr. Gajendra Prasad Das for helping with statistics.

Funding: No funding sources Conflict of interest: None declared

Ethical approval: Not required

\section{REFERENCES}

1. Carr DB, Gabbe S. Gestational Diabetes: Detection, Management, and Implications. Clin Diabetes. 1998;16(1):4.

2. Langer O, Brustman L, Anyaegbunam A, Mazze R. The significance of one abnormal glucose tolerance test value on adverse outcome in pregnancy. Am J Obstet Gynecol. 1987;157:758-63.

3. American Diabetes Association. Standards of medical care in Diabetes-2013. Diabetes Care. 2013;36(Suppl 1):S11-66.
4. Seshiah V, Balaji V, Balaji MS, Sanjeevi CB, Green A. Gestational diabetes mellitus in India. J Assoc Physicians India. 2004;52:707-11.

5. Naylor CD, Sermer M, Chen E, Sykora K. Cesarean delivery in relation to birth weight and gestational glucose tolerance. Pathophysiology or practice style? JAMA. 1996;275:1165-70.

6. Deerochanawong C, Putiyanun C, Wongsuryrat M, Serirat S, Jinayon P. Comparison ofNational Diabetes Data Group and World Health Organization criteria for detecting gestational diabetes mellitus. Diabetologia. 1996;39(9):1070-3.

7. Schmidt MI, Duncan BB, Reichelt AJ, Branchtein L, Matos MC, Costae Forti A et al. Gestational diabetes mellitus diagnosed with a2-h75-goralglucose tolerance test and adverse pregnancy outcomes. Diabetes Care. 2001;24(7):1151-5.

8. Odar E, Wandabwa J, Kiondo P. Maternal and fetal outcome of gestational diabetes mellitus in Mulago Hospital, Uganda. Afr Health Sci. 2004;4(I):9-14.

9. Crowther CA, Hiller JE, Moss JR, Mc Phee AJ, Jeffries WS, Robinson J. Effect of treatment of gestational diabetes mellitus on pregnancy outcomes. N Engl J Med. 2005;352(24):2477-86.

Cite this article as: Das A. Maternal and perinatal outcomes in diabetic pregnancies at BPKIHS. Int J Reprod Contracept Obstet Gynecol 2017;6:2262-6. 